\title{
MANEJO DA DOR PEDIÁTRICA: PROJEÇÕES E PERSPECTIVAS DA EQUIPE DE ENFERMAGEM
}

\author{
Stela Faccioli Ederli ${ }^{1}$, Berta Aparecida de Oliveira Bazote ${ }^{1}$, Lucas Lima de Moraes ${ }^{1}$, Maria Eduarda de Souza \\ Aredes $^{1}$, Mauren Teresa Grubisich Mendes Tacla ${ }^{2}$ \\ ${ }^{1}$ Universidade do Oeste Paulista - UNOESTE, Presidente Prudente, SP. ${ }^{2}$ Universidade Estadual de Londrina - UEL, \\ Londrina, PR. e-mail: stelafaccioli@hotmail.com
}

\section{RESUMO}

O objetivo deste estudo foi compreender os critérios utilizados pela equipe de enfermagem para avaliar a dor da criança hospitalizada em uma UTIP. Trata-se de um estudo qualitativo, tendo como público alvo a equipe de Enfermagem de uma UTIP de um hospital do interior do Estado de São Paulo. Foram realizados grupos focais alternando em dias ímpares e pares. Analisou-se os dados utilizando Análise Temática proposta por Bardin. A análise resultou em duas categorias, sendo a primeira: "Vivenciar a multidimensionalidade da dor da criança: projeções e sentimentos" e a segunda: "Avaliação da dor pediátrica: um cuidado a ser aprimorado". Portando as entrevistadas compreendem de forma subjetiva o conceito de dor e que as mesmas não utilizam critérios padronizados para a avaliação da dor, o que gera uma assistência não sistematizada diante do manejo da dor da criança hospitalizada.

Palavras-chave: dor, unidades de terapia intensiva pediátrica, enfermagem, medição da dor, criança.

\section{PEDIATRIC PAIN MANAGEMENT: PROJECTIONS AND PERSPECTIVES OF THE NURSING STAFF}

\section{ABSTRACT}

The objective of the study was to understand the criteria used by the nursing team to assess the pain of children hospitalized in a PICU. This is a qualitative study, targeting the Nursing team of a PICU at an inland hospital of the State of São Paulo. Focus groups were held alternating on odd and even days. The data was analyzed using Thematic Analysis proposed by Bardin. The analysis resulted in two categories, the first: "Experiencing the multidimensionality of children's pain: projections and feelings" and the second: "Pediatric pain assessment: care to be improved". Therefore, the interviewees understand the concept of pain in a specific way and do not use standardized criteria for pain assessment, which generates nonsystematic assistance in the management of pain in hospitalized children.

Keywords: pain, pediatric intensive care unit, nurse, pain measurement, child.

\section{INTRODUÇÃO}

A Unidade de Terapia Intensiva Pediátrica (UTIP) é caracterizada por admitir crianças e adolescentes com uma ou mais disfunções orgânicas podendo ser classificados como criticamente enfermos. Além disso, o ambiente da UTI pediátrica pode produzir ansiedade e dor causadas por procedimentos rotineiros e invasivos. Nessa perspectiva é importante assegurar aos mesmos o direito à sobrevida e a uma assistência humanizada, bem como, exposição mínima aos riscos decorrentes do tratamento e monitoramento permanente da evolução do tratamento, assim como de seus efeitos adversos ${ }^{1}$.

Segundo Knobel $^{2}$ o paciente pediátrico grave pode ser compreendido como aquele que apresenta um acelerado processo patológico com a presença de dor na maioria das vezes e necessita de atitudes decisivas dos profissionais para garantir a assistência à saúde apropriado a sua condição clínica. 
Considerando essa população, pode-se afirmar a vulnerabilidade e exposição a diversos procedimentos, tais como inserções intravenosas repetidas, coleta de exames laboratoriais por meio da punção venosa, próteses e cirurgias. Diante disso, a dor pode ser um problema real e significativo dentro das UTIP ${ }^{3}$.

A dor é um dos sintomas comuns nas diferentes enfermidades sendo descrita pela Associação internacional para o Estudo da Dor (IASP) como: "Uma experiência sensorial e emocional indesejada associada com lesão real ou potencial do tecido, ou descrita em termos de tal dano" ${ }^{\prime 4}$.

Sendo considerada um fenômeno multidimensional, a dor possui componentes sensoriais, fisiológicos, cognitivos, afetivos, comportamentais e espirituais. Tais componentes alteram a forma como a dor é experimentada, modificando a transmissão dos estímulos ao cérebro ${ }^{5}$. Podendo ser classificada desde dor leve e inconsequente ao tipo intolerável e persistente ${ }^{6}$.

Da mesma maneira que os adultos, as crianças podem sofrer vários tipos de dor, sendo estes relacionados à doença, trauma, fator psicológico ou mesmo de etiologia desconhecida ${ }^{7}$.

O tratamento e alívio da dor é direito da criança e deve ser considerada sua faixa etária e seu nível cognitivo, visto que os autorrelatos frente às queixas de dor podem ser um indicativo importante e fidedigno de um episódio de dor. Além disso, a crença de que crianças não respondem e nem possuem lembranças das experiências dolorosas anteriores é falsa ${ }^{8}$.

E é fundamental que a dor em crianças seja adequadamente identificada, avaliada e, sobretudo, tratada, o que ainda é uma tarefa complexa para a equipe de saúde ${ }^{9}$.

Reconhecer e avaliar a dor com precisão são habilidades fundamentais para seu manejo. No entanto, ao pensar nas crianças hospitalizadas nas UTIP, a administração da sedação e a capacidade verbal limitada das crianças podem dificultar a avaliação dolorosa ${ }^{10}$.

A sedação é um mecanismo de retardar ou inibir as respostas neurossensoriais como, por exemplo, nos casos de inibição a sedação profunda proporcionando assim ao paciente um quadro de tranquilidade e vigília. Já a analgesia é utilizada para interromper ou amenizar o processo de dor proporcionando assim uma melhor qualidade em sua reabilitação ${ }^{11}$.
No ambiente de Terapia Intensiva Pediátrica, os analgésicos e sedativos estão presentes rotineiramente devido à gravidade dos diagnósticos e a múltipla realização de intervenções, procedimentos invasivos, necessidade de suporte ventilatório, além dos efeitos adversos de agitação, ansiedade e ambiente hostil para criança ${ }^{12}$.

Pensando na correlação entre dor, analgesia e sedação estudos evidenciam que a dor em praticamente todos os serviços de saúde são subdiagnosticada, mal avaliadas ou negligenciadas dificultando 0 processo de avaliação e recuperação do paciente ${ }^{13}$.

A Joint Commission on Accreditation of Healthcare Organizations (JCAHO) ${ }^{14}$ descreve a dor como sendo o 50 sinal vital, devendo ser avaliada e registrada pela equipe de enfermagem junto aos demais sinais vitais, para que condutas possam ser tomadas e resultados avaliados ${ }^{6,14}$. A avaliação da dor é necessária antes de procedimentos dolorosos para determinar a condição da criança e, após o procedimento, para verificar a eficácia das estratégias adotadas para seu manejo ${ }^{15}$.

Quando tratamos de métodos de avaliação da dor podemos citar medições fisiológicas, medições comportamentais, auto relatos e instrumentos multidimensionais apropriados para cada criança, para que possamos melhor avaliar as manifestações de dor ${ }^{8}$.

Diante do manejo adequado da dor pediátrica, o tratamento torna-se possível, evitando medidas farmacológicas desnecessárias e/ou possibilitando 0 tratamento não farmacológico para alívio da dor. Evitar a dor de uma criança deve ser uma obrigação ética e humanitária, além de um direito previsto em lei. Faz-se necessário, portanto, que o alívio da dor na criança se torne uma prioridade para todos os profissionais de saúde ${ }^{16}$.

Frente a isso, o presente estudo teve como objetivo identificar a percepção da equipe de enfermagem frente a dor da criança hospitalizada em uma UTIP. A pesquisa teve como questão norteadora "Quais os sentimentos vivenciados pela equipe de enfermagem frente a criança gravemente enferma, hospitalizada e com dor?"

\section{METODOLOGIA}

Estudo qualitativo, realizado em uma Unidade de Terapia Intensiva Pediátrica (UTIP) de 
um hospital do interior do Estado, no Oeste Paulista. Optou-se pela pesquisa qualitativa pois permite a abordagem compreensiva diante da diversidade dos pontos de vistas dos diferentes atores estudados.

Participaram do estudo a equipe de enfermagem atuante na UTIP. Justifica-se a escolha do público alvo em razão de prestarem assistência direta às crianças hospitalizadas, sendo os responsáveis pela verificação dos sinais vitais, incluindo a dor. A população de estudo foi selecionada em sua totalidade, exceto, os que estiverem em período de férias ou licença no prazo pré-estabelecido à coleta de dados.

Para o desenvolvimento dos grupos focais, os pesquisadores convidaram os técnicos de enfermagem e enfermeiros na UTIP, apresentando objetivo e interesses do estudo. Após o levantamento dos interessados, foi feito um novo convite pessoalmente, por e-mail e por telefone, informando data e local da atividade.

Os grupos focais foram realizados em salas de uma instituição privada vinculada ao hospital em questão, para que garantisse a privacidade dos participantes e para garantir o rigor metodológico preconizado nos ambientes dos grupos focais. Foram realizados grupos focais alternando em dias ímpares e pares para que pudesse abranger o maior número de participantes.

No dia de cada grupo focal, estavam presentes os três autores principais, sendo dois no papel de observadores que junto com a docente orientadora fizeram anotações para apoio às transcrições dos grupos. Foi realizada apenas uma sessão de grupo focal por turno com a participação média de cinco funcionárias, considerando a saturação de dados empíricos, totalizou 16 participantes.

Para aproximação ao tema, utilizou-se como disparador imagens relacionadas a dor pediátrica e hospitalização infantil, sendo dispostas frente aos participantes para que todos pudessem ter acesso as mesmas. A questão norteadora foi: "Fale-me o que vem em sua mente quando você pensa na dor infantil" e foram utilizadas questões acessórias para aprofundar a discussão: Como é para você a vivência de prestar cuidado a uma criança com dor? Como você avalia a dor da criança?

Para finalizar os grupos, os pesquisadores retomaram os pontos principais discutidos em grupo para que os participantes pudessem reformular ou acrescentar as colocações. As discussões foram gravadas com o consentimento dos participantes e cada grupo durou de 60 a 90 minutos.

Em caso de aceite os entrevistados assinaram o Termo de Consentimento Livre e Esclarecido, cumprindo assim os princípios éticos e morais. Após o aceite, as discussões foram gravadas por meio de um gravador digital, transcritas e posteriormente excluídas. Com o intuito de garantir o sigilo frente a identidade dos participantes, no início do grupo, foram entregues crachás com nomenclaturas de flores e frutas e não foram divulgadas informações de caráter pessoal.

Para a análise dos dados foi utilizada a Análise de Conteúdo Temática proposta por Bardin, $2011^{17}$ seguindo as etapas propostas: a pré-análise que consiste na leitura flutuante, o primeiro contato com os textos, captando o conteúdo genericamente. A segunda etapa denominada de exploração do material que consistiu no desmembramento do texto em unidades/categorias, reagrupamento por categorias para análise posterior - do material. Por fim, a terceira etapa, o tratamento dos dados e a interpretação, onde o pesquisador fará inferências com abordagem qualitativa trabalhando com significações ${ }^{18}$.

0 presente estudo foi submetido ao Comitê de Ética em Pesquisa, seguindo a Resolução n. 466/2012 que dispõe sobre as normas regulamentadoras das pesquisas que envolvem seres humanos com parecer favorável e CAAE no 86584218.8.0000.5515.

\section{RESULTADOS}

Percebeu-se que as entrevistadas atribuíram diversas dimensões e percepções a definição da dor, considerando-a como desconforto físico, prejudicial e emocional. Salientaram ainda que a dor na criança a torna irritada e que pode desestabilizar o quadro de saúde apresentado.

As entrevistadas fazem uma associação com os mediadores químicos e receptores relacionados ao estímulo doloroso, porém, também acreditam que o estímulo pode ser sentimental.

"Qualquer tipo de desconforto que prejudique a criança de alguma forma como dispositivo, manipulação, qualquer tipo de desconforto que causa dor né e prejudica a criança na minha opinião é dor". Maçã 
"As vezes a dor não é só você está sentindo a dor né? dor sentimental e tudo mais".

"Carência também gera dor, sentimento". Pêra

"Dor é uma coisa que incomoda, que irrita, que desestabiliza". Abacaxi

"É uma manifestação do organismo referente a um estimulo no caso doloroso, tem várias formas de sentir dor que é química, térmica esses mediadores químicos são, estão geralmente localizados na superfície da pele $e$ eles são encaminhados ao cérebro através da medula e os receptores recebem e ai isso causa a sensação de dor". Lavanda

Quando questionadas sobre os sentimentos em relação as crianças hospitalizadas, as entrevistadas referem dificuldade em assistir as crianças com dor devido as projeções com seus filhos. Referem relacionar os momentos de tristeza e sofrimento das crianças hospitalizadas com seus filhos e essa projeção torna o cuidado mais complexo.

"Ao mesmo tempo em que é difícil pra quem tem filhos eu acho que é mais difícil ainda né que a gente acaba associando". Jasmim

"A gente que é mãe a gente fala poderia ser um filho nosso em cima daquele leito né então assim é tem períodos de tristeza". Graviola

"e você vê tanta coisa aqui que o filho tosse na casa aí você fala vai ser uma TB você só pensa no pior, você vai pirando". Melancia

Ainda no âmbito dos sentimentos e projeções, as entrevistadas referiram sofrimento maior em assistir uma criança hospitalizada do que um adulto, pois justificam que além da dor propriamente dita, o choro da criança permeia o medo e a agonia de estar ali e que em muitos casos, mesmo após as medidas farmacológicas, o choro permanece.

"Por que você sofre muito mais com uma criança do que com um adulto. Você faz o tratamento do adulto, mas a criança mexe mais com o emocional da gente e você vendo a criança agonizada por que essa daqui ta com medo de uma punção, ta chorando com dor ta com medo de tudo, então você tem que ter todo um trabalho psicológico com a criança e com a mãe". Lavanda

"A gente tem dó né? Preocupação porque você quer o bem né? Você quer tirar aquela dor as vezes porque as vezes você faz uma medicação para dor e ainda permanece com dor dependendo da patologia do caso que é". Joaninha
"Se você ver que ela está com dor você nem consegue trabalhar você fica ali tentando fazer alguma coisa". Leão

Sobre a dor pediátrica, um anseio constante de profissionais que atuam com crianças hospitalizadas é o de aliviar a dor, porém, para que esse alívio seja possível, é necessária uma avaliação fidedigna. Diante disso, as entrevistadas atribuíram essencialidade ao momento de avaliação, enfatizando a importância de não deixar uma criança sentir dor estando dentro de uma unidade hospitalar.

As falas também permearam a necessidade do alívio da dor para que a assistência a criança possa ser prestada da maneira adequada, visto que, uma criança com dor impossibilita o cuidado, além do desconforto em ser manipulada.

"Porque a pessoa já está em um hospital em primeiro lugar o hospital é um lugar que tem que oferecer conforto e pra ter dor dentro de um hospital, só se não for diagnosticada avaliada $e$ recebido o tratamento devido". Abacaxi

"Precisa avaliar o tipo de dor e tomar as providencias e não ficar tacando remédio né mascarando". Pera

"Uma criança com dor não deixa você fazer nada, uma criança com dor ela não deixa você nem aferir a pressão, você não consegue nem chegar perto dela, então para você conseguir ter os cuidados, verificar sinais você precisa tratar a dor porque criança com dor não deixa". Lavanda

"Ninguém merece dentro de um hospital ter dor, qualquer tipo de paciente, vamos supor, tem gente que vê e diz que é chilique, não cabe a mim esse julgamento, então se você está com dor, o máximo que eu posso fazer é te amenizar disso, não é justo, não é humano você estar dentro de um hospital e estar com dor, então eu não posso julgar sua dor". Melancia

Atualmente, devido a sua importância e multidimensionalidade, a dor é considerada como quinto sinal vital pela Organização Mundial de Saúde, porém, ainda não é considerada dessa forma em muitas instituições, como a unidade em questão.

Para a avaliação da dor, os profissionais tendem a usar critérios próprios que vão de encontro com fatores intrínsecos de cada profissional ou extrínsecos relacionados ao ambiente de trabalho e rotina institucional. Porém, a avaliação da dor é um processo 
complexo e necessita de instrumentos padronizados e validados para cada faixa etária.

Os entrevistados referiram utilizar critérios para avaliação da dor como expressões faciais, choros e gritos, alterações de sinais vitais e gemência. Sendo assim, cada profissional avalia de acordo com a sua percepção a partir dos sinais apresentados por cada criança, não utilizando escalas.

"Ah a gente pede pra criança colocar a mão, a criança coloca a mão onde dói, que nem a criança fez cirurgia e tá chorando, eu tenho certeza que é dor aquilo ali". Ameixa

"Até na hora de dormir o sussurro dela é diferente e você sabe que ela tá com dor, as vezes ela fica cansada, ela está inquieta não está dormindo tranquila". Pêssego

"Vê o aspecto da criança vê a face, a fisionomia é diferente de uma dor de manhã, de uma dor porque a mãe saiu e foi comer, por que as vezes tem fome é diferente de uma dor que é dor mesmo, a dor que é dor a criança geme, a fisionomia fica abatido é diferente". Maça

"É pela fisionomia, pelo choro a criança as vezes fica agitada quando está com dor. Pressão sobe, da taquicardia criança fica desconfortável". Graviola

No contexto da criança hospitalizada, temos ainda as crianças que estão ou são impossibilitadas de praticar o auto relato, o que dificulta a avaliação da dor, pois elas não conseguem relatar o que sentem ou a localização da mesma. Diante disso, as entrevistadas referiram avaliar a dor nessas circunstâncias levando em consideração os sinais vitais e a experiência profissional que trazem consigo.

"É porque uma criança que está intubada você vê que está gemente ta com dor, mas você não sabe onde, se ela tem uma cirurgia a gente deduz que é a cirurgia, mas pode ser a cabeça né, pode ser a posição que está o braço né". Pêra

"Principalmente quando está intubado que não demonstra aí se você sente dor a frequência cardíaca vai lá em cima ou pode dar temperatura alta também". Leão

"Você não sabe se ela está com dor ou não, então é só pelos sinais mesmo". Ameixa

\section{DISCUSSÃO}

A dor pode ser considerada uma experiência sensitiva e emocional podendo estar relacionada a um dano tecidual ou possível dano, ainda, a experiência dolorosa leva a complicações físicas e emocionais resultando em aumento da permanência hospitalar, bem como, elucidado por este estudo em que as entrevistadas relatam a dor como carência e outros aspectos sentimentais, além de correlacionar com aspectos fisiológicos do processo de instalação da dor $^{19}$.

Aspectos como a projeção do cuidado permeiam as falas das entrevistadas demonstrando a dificuldade em comunicar a dor socialmente evidenciando sofrimento quando o tema é cuidar de crianças com dor, devido as manifestações como choro, agonia e gemência fazendo com que projetem o cuidado aos seus familiares, bem como elucidado por Karos et al. ${ }^{20}$ que aborda a dificuldade dos profissionais em lidar socialmente com a dor da criança.

O cuidado infantil hospitalar para os profissionais de enfermagem é citado como alto grau de sofrimento psíquico uma vez que eles são dominados por sentimentos de instabilidade emocional devido ao convívio com o cuidado à criança em consonância com a vivência do sofrimento familiar, dados esses que corroboram com o presente estudo uma vez que as entrevistadas relatam dificuldade e angustia no processo de cuidar das crianças gravemente enfermas $^{21}$.

Os sentimentos supracitados podem ser adquiridos por meio da forma de manifestação do processo de dor na criança hospitalizada em uma unidade de terapia intensiva, bem como, o cenário por tratar-se de uma rotina intensiva e ininterrupta de cuidado. Durante as entrevistas surgiram falas que as colocam em obrigatoriedade de fornecer conforto e alívio da dor por tratar-se de uma instituição hospitalar, assim retificando os aspectos psicológicos prejudiciais aos profissionais que cuidam ${ }^{22}$.

Por anseio de confortar o paciente hospitalizado, diante a situação de vulnerabilidade à qual estão submetidos surge o uso exacerbado da terapia farmacológica para o alivio da dor, aspectos esses que contradizem a percepção dos profissionais de enfermagem podendo ser observada pela seguinte fala "Precisa avaliar o tipo e tomar as providências e não ficar tacando remédio né mascarando". Aspecto este também observado por Batalha et al. ${ }^{23}$ que reconhece a negligência da avaliação da dor como prejudicial para o cuidado da criança hospitalizada.

Observado nas entrevistas, o cuidar de uma criança com dor representa uma barreira para efetividade do cuidado, isto, pois as 
manifestações de dor impossibilitam cuidados básicos como aferir os sinais vitais, realizar troca de decúbito, dificuldades estas também observadas por Santos e Maranhão ${ }^{24}$ além de relatar que a avaliação da dor é uma grande dificuldade enfrentada pelas equipes de enfermagem.

Avaliar a dor infantil exige uma ampla visão e embasamento na multidimensionalidade uma vez que aspectos subjetivos podem imperar no momento da avaliação, instituir-se uma escala de mensuração torna-se de suma importância no que tange a assistência de enfermagem qualificada ao usuário ${ }^{25,26}$, aspectos como estes podem ser observados pelas falas das entrevistadas que relatam avaliar a dor de forma subjetiva colocando em questionamento a qualidade da assistência.

Dificuldades como mensuração da dor em pacientes que estão impossibilitados de autor relatar a dor podem ser observados nesse estudo, evidenciando a necessidade de implementação de instrumentos que abordem os aspectos multidimensionais da dor, tornando o manejo da dor mais fidedigno e a assistência mais segura e eficaz ao paciente a qual vivencia a experiência dolorosa. Implementar escalas são medidas de grande importância para prática profissional, pois norteiam e sistematizam a forma de manejar a dor no infante.

\section{CONCLUSÃO}

Considera-se que as entrevistadas compreendem parcialmente o conceito de dor tornando-a subjetiva e inteiramente relacionada a aspectos emocionais, bem como, utilizam da subjetividade para avaliar a dor das crianças hospitalizadas fazendo com que a assistência não seja sistematizada. Sendo assim torna-se valioso explorar mais a temática no conceito acadêmico para que possamos formar profissionais ainda mais capacitados para atuar no contexto dor em consonância à implantação de escalas de avaliação da dor nos serviços de enfermagem para nortear, qualificar e sistematizar o cuidado intensivo pediátrico.

\section{CONFLITO DE INTERESSES}

Os autores declaram não haver qualquer potencial conflito de interesse que possa interferir na imparcialidade deste trabalho científico.

\section{REFERÊNCIAS}

1. Dantas LVRP, Dantas TSP, Santana Filho VJ, Azevedo-Santos IF, Santana JM. Pain assessment during blood collection from sedated and mechanically ventilated children. Rev Bras Ter Intensiva. 2016;28(1):49-54. DOI: http://dx.doi.org/10.5935/0103-507X.20160013

2. Knobel E. Condutas no paciente grave. 4.ed. São Paulo: Atheneu; 2016.

3. Breau LM, Burkitt C. Assessing pain in children with intellectual disabilities. Pain Res Manag. 2009;14(2):116-20.

https://doi.org/10.1155/2009/642352

4. Blasi DG, Candido LK, Tacla MTGM, Ferrari RAP. Avaliação e manejo da dor na criança: percepção da equipe de enfermagem. Semina: Ciên Biol Saúde. 2015;36(1):301-10.

https://doi.org/10.5433/16790367.2015v36n1Suplp301

5. World Health Organization. Who guidelines on the pharmacological treatment of persisting pain in children with medical illnesses. Geneva: World Health Organization; 2012.

6. Silva JA. The challenge of pain. Physchol Neurosci. 2014;7(1):1-2. DOI: http://dx.doi.org/10.5935/1806-0013.2017012

7. Serrano SC, Barbosa SMM, Queiroz EJ, Campos FG, Santos ASV. Peculiarities of neuropathic pain in children. Rev Dor. 2016;17(1):110-2. DOI: http://dx.doi.org/10.5935/1806-0013.20160061

8. Krauss BS, Calligaris L, Green SM, Barbi E. Current concepts in management of pain in children in the emergency department. Lancet. 2016;387(10013):83-92.

DOI: http://dx.doi.org/10.1016/S0140-6736(14)61686$\underline{x}$

9. Gonçalves B, Holz AW, Lange C, Maagh SB, Pires CG, Brazil CM. Care of children in pain admitted to a pediatric emergency and urgency unit. Rev Dor. 2013;14(3)179-83. DOI: http://dx.doi.org/10.1590/S180600132013000300005

10. Mitchell AJ, Yates CC, Williams DK, Chang JY, Hall RW. Does daily kangaroo care provide sustained pain and stress relief in preterm 
infants?. J Neonatal Perinatal Med. 2013;6(1):4552. https://doi.org/10.3233/NPM-1364212

11. Miranda ML, Bersot CDA, Villela N.R. Sedação, analgesia e bloqueio neuromuscular na unidade de terapia intensiva. Rev Hosp Univ Pedro Ernesto. 2013;12(3):102-9. DOI: http://dx.doi.org/10.12957/rhupe.2013.7537

12. Monitto CL, Hsu A, Gao S, Vozzo PT, Park OS, Roter $D$ et al. Opioid prescribing for the treatment of acute pain in children on hospital discharge.

Anesthesia-Analgesia. 2017;125(6):2113-22.

DOI: http://dx.doi.org/10.1213/ANE.00000000000025 $\underline{86}$

13. Ribeiro SF, Pinto JCP, Ribeiro JB, Felix MMS, Barroso SM, Oliveira LF et al. Pain Management at Inpatient Wards of a University Hospital. Rev Bras Anestesiol. 2012;62(5):599-611. https://doi.org/10.1016/S0034-7094(12)70160-5

14. Joint Commission International Accreditation Standards for Hospitals. $2^{\text {nd }}$. ed. Illinois, 2004.

15. Lee GY, Yamada J, Kyololo O`B, Shorkey A, Stevens B. Pediatric clinical practice guidelines for acute procedural pain: a systematic review. Pediatrics. 2014;133(3):500-15. DOI: http://dx.doi.org/10.1542/peds.2013-2744

16. Candido LK, Tacla MTGM. Avaliação e caracterização da dor na criança: utilização de indicadores de qualidade. Rev Enfer UERJ. 2015;23(4):526-32. DOI: http://dx.doi.org/10.12957/reuerj.2015.10514

17. Bardin L. Análise de conteúdo. São Paulo: Edições 70; 2011, p.229.

18. Minayo MCS, Deslandes SF, Gomes R. Pesquisa social: teoria, método e criatividade. 30.ed. Petrópolis: Vozes; 2016.

19. Silva EM, Olinda AG, Barros FHG, Silva LO et al. A percepção da equipe de enfermagem na avaliação da dor em unidades pediátricas: revisão sistemática da literatura. Rev Mult Psic. 2018;12(42):777-89.

https://doi.org/10.14295/idonline.v12i42.1536

20. Karos K, Williams ACC, Meulders A, Vlaeyen JWS. Pain as a threat to the social self: a motivational account. Pain. 2018;159(9):1690-5. DOI:

http://dx.doi.org/10.1097/j.pain.0000000000001 $\underline{257}$

21. Campos GRP, Passos MAN. Sentimentos da equipe de enfermagem decorrentes do trabalho com crianças em uma unidade de queimados. Rev Bras Queimaduras 2016;15(1):35-41.

22. Silva EM, Olinda AG, Barros FHG, Silva LO. et al. A Percepção da Equipe de Enfermagem na Avaliação da Dor em Unidades Pediátricas: Revisão Sistemática da Literatura. Rev. Mult. Psic. 2018;12(42):777-789.

https://doi.org/10.14295/idonline.v12i42.1536

23. Batalha LMC, Sousa AFD. Autoavaliação da intensidade da dor: correlação entre crianças, pais e enfermeiros. Rev Enf Ref. 2018;4(17):1522. DOI: http://dx.doi.org/10.12707/RIV18002

24. Santos JP, Maranhão DG. Cuidado de Enfermagem e manejo da dor em crianças hospitalizadas: pesquisa bibliográfica. Rev Soc Bras Enferm Ped. 2016;16(1):44-50. https://doi.org/10.12957/reueri.2017.15379

25. Stevens B, Franck L, Gibbins S, et al. Determining the structure of acute pain responses in vulnerable neonates. Can J Nurs Res. 2007;39(2):32-47.

26. Stevens B, Johnston C, Petryshen P, Taddio A. Premature infant pain profile: development and initial validation. Clin J Pain. 1996;12(1):13-22. https://doi.org/10.1097/00002508-199603000$\underline{00004}$ 